\title{
Development of a score based on urinalysis to improve the management of urinary tract infection in children
}

\author{
Mohamed Hamed Bahbah ${ }^{1}$, Ahmed Anower Khattab ${ }^{1}$, Rania Salah El Zayat ${ }^{1}$, \\ Abdelwahab Mohamed Badr ${ }^{1,2, *}$ \\ ${ }^{1}$ Pediatrics department, Faculty of Medicine, Menoufia University, Shebin El-Kom, Menoufia, Egypt \\ ${ }^{2}$ Pediatrics resident, Shebien Elkom Teaching Hospital, Menoufia, Egypt
}

Email address:

houbabadr@outlook.com (A. M. Badr), raniaelzayat1@yahoo.com (R. S. El Zayat)

To cite this article:

Mohamed Hamed Bahbah, Ahmed Anower Khattab, Rania Salah El Zayat, Abdelwahab Mohamed Badr. Development of a Score Based on Urinalysis to Improve the Management of Urinary Tract Infection in Children. American Journal of BioScience.

Vol. 3, No. 1, 2015, pp. 1-6. doi: 10.11648/j.ajbio.20150301.11

\begin{abstract}
Objectives: Our aim is to develop a score that may help in reducing the misuse of antibiotics in treatment of urinary tract infection before appearance of urine culture. Background: The need for reducing unnecessary antibiotic treatment is being emphasized in the management of urinary tract infections (UTI), a disease frequent in childhood. An ideal test should provide early diagnosis without the waiting times of urine culture, but even a simple test of exclusion could significantly improve patient management. Methods: We evaluated the sensitivity, specificity, negative and positive predictive value of urine analysis, dipstick (leukocyte esterase and nitrite) and bacterial colony count in children with suspected UTI. Multivariable logistic regression analysis was used to identify the set of variables that best predict positive culture results and develop a numerical risk score. Results: Of 200 children were having symptoms of UTI, 93 child had confirmed UTI with prevalence $46.5 \%$. Parameters significantly associated with the presence of infection in multivariable analysis were age 9-12 years $(\mathrm{p}<$ 0.05 ), leukocyte esterase positive ( $p<0.001)$, nitrite positive ( $p<0.05)$, pus cell $>10 / \mathrm{HPF}(\mathrm{p}<0.05), \operatorname{RBCs}>10 / \mathrm{HPF}(\mathrm{p}<0.05)$ and bacteria $\geq 10^{\wedge} 6(\mathrm{p}<0.05)$.The derived score ranged from 1 to 7 , with higher values indicating higher risk of UTI. Conclusion: This routine method could improve the management of UTI in children by early identifying patients with low probability of infection, for which antibiotic treatment can be withheld until the results of urine culture become available.
\end{abstract}

Keywords: Urinary Tract Infection, Urinalysis

\section{Introduction}

Urinary tract infection (UTI) is one of the most common bacterial infections in infants and children and the most common bacterial infection in infants under three months. (19).

Although the diagnosis and treatment of urinary tract infections UTI are relatively straightforward, a large proportion of the population may be unnecessarily exposed to antibiotics while waiting for the results of urine culture, with implications for costs and antibiotic resistance. (12).

In infants and young children, additional issues include the severity and/or lack of specificity of symptoms, the higher risk of side effects of antibiotics, and the possibility of kidney damage in case of inappropriate treatment. (3).Culture of urine is the reference standard for the diagnosis of UTI. (23).
Continuing efforts are spent in searching for alternative, more rapid and still accurate tests. This is especially important in children, since both a delay in treatment and the inappropriate administration of antibiotics can result in potentially serious adverse effects. (21).

\section{Patients \& Methods}

\subsection{Patients}

Two hundreds of children of both gender 91 males (45\%) and 109 females $(54 \%), \leq 15$ years old, born and living in Menoufiya governorate and attended to outpatient clinic of Berket Al Sabaa Hospital complaining of symptoms of UTI were included in this study. 
This study was done from March 2013 to March 2014.

\subsubsection{Inclusion Criteria}

Children were selected in random way considered legible for the study as they met the following criteria:

1. Age $<15$ years old.

2. Children having symptoms of urinary tract infection e.g. (dysuria, frequency and urgency)

\subsubsection{Exclusion Criteria}

1. Children under treatment of antibiotic.

2. Children have history of chronic renal disease.

An explanation of the purpose of the study to parents of children included in the study was done and consent was taken.

\subsection{Methods}

\subsubsection{All the 200 Children Included in the Study were} Subjected to the Following

A. Full history taking.

B. Complete clinical examination.

C. Urine specimens collected then the following analysis were done on urine specimens:

- $\quad$ Routine urine analysis.

- Dipstick analysis for leucocyte esterase and nitrite.

- Bacterial colony count.

- Urine culture.

Table 1. shows the formulation of the scoring system.

\begin{tabular}{lll}
\hline & The range & The score \\
\hline \multirow{2}{*}{ Age } & $1-4$ years & 2 \\
& $>4-9$ years & 1 \\
& $>9-14$ years & 0 \\
& $(0-10)$ & 0 \\
Pus cells & $(>10-<100)$ & 1 \\
& $(\geq 100)$ & 2 \\
RBCs & $(0-10)$ & 0 \\
& $(>10)$ & 1 \\
Leukocyte esterase & $(-\mathrm{VE})$ & 0 \\
& $(+\mathrm{VE})$ & 1 \\
Nitrates & $(-\mathrm{VE})$ & 0 \\
& $(+\mathrm{VE})$ & 1 \\
Bacterial colony count & $(0-10000)$ & 0 \\
& $(>10000-$ & 1 \\
& $<100.000)$ & 2 \\
\hline
\end{tabular}

All investigations were made in outpatient laboratory.

The following table shows the formulation of our scoring system as age will be given a score as follow: children from 1-4 years will be given score 2, children from 4-9 years will be given a score 1, while children from 9-14 years will be given a score 0 . According to pus cell cases that had (0-10) will be given 0 , while cases that had (10-100) will be given 1 , and cases that had (over 100) will be given 2. Concerned to
RBCs cases who had (0-10) will be given 0 , while cases that had (more than 10) will be given 1. According to BCC cases who had (0-10000) will be given 0 , cases who had (10000100000) will be given 1 , and cases who had (more than 100000) will be given 2. Concerned to LE negative cases will be given 0 , while LE positive cases will be given 1 . Concerned to nitrite negative cases will be given 0 , while nitrite positive cases will be given 1 .

\subsubsection{Collection of Urine Samples}

When collecting urine samples, two things must be taken in accounts, the way of collecting urine and the time until the sample is tested (7).

According to NIHCE a midstream clean catch urine sample is the recommended method for urine collection. Urine samples must be tested immediately after collection, but if urine cannot be tested and cultured within 4 hours of collection, the sample should be refrigerated or preserved with boric acid (16).

Urine can be stored in the refrigerator for up to 24 hours (20).

\subsubsection{The Investigations}

\subsubsection{Microscopic Urine Examination}

Urine samples from children were taken for analysis by microscopic examination. Urine samples were collected into $200-\mathrm{ml}$ vessel, $10 \mathrm{ml}$ of urine collected was centrifuged in a plastic centrifuge tube. Pouring off most of the supernatant and resuspend the sediment in the remaining supernatant. Examining of one drop of the suspension (on slide) under a light microscope. Red blood cell count or WBC of 10/HPF or more reveals the presence of hematuria or pyurea.

\subsubsection{Dipstick Analysis}

Results of dipstick (Medi-Test, Combi 10 SGL) were interpreted visually according to standard color charts:

The leukocyte esterase (LE):

This normally suggests inflammation of some sort, often from a urinary tract infection. A simple dipstick test as part of a urinalysis can detect the presence of this enzyme (9).

This test reveals the presence of granulocyte esterases. The esterases cleave a derivatizedpyrazole amino acid ester to liberate derivatizedhydroxypyrazole.Thispyrazole then reacts with a diazonium salt to produce a purple color. (9).

The nitrite:

It is based on the ability of pathogens to reduce nitrate to nitrite. It has sensitivity $60 \%$ although the specificity is $100 \%$ (2).

Normal test is negative.

Any degree of uniform pink to red color that appears on the test confirms the presence of nitrite, and indicates a significant bacteriuiria. The color intensity is not significant, and is not proportional to the number of bacteria present in the urine sample.

Pink spots or pink edges were interpreted as a negative result.

Urinary retention in the bladder (at least 4-8 hours) and 
antibiotics were discontinued for at least 3 days before the test was performed to obtain an accurate result.

\subsubsection{Bacterial Colony Counting}

The number of colonies is multiplied by the appropriate factor to give the colony count per mL urine. (17).

The presence of bacteria in a fresh, Gram-stained specimen of uncentrifuged urine correlates with 105 CFUs per $\mathrm{mL}$ in culture. An "enhanced urinalysis," combining the counting chamber assessment of pyuria noted previously with Gram staining of drops of uncentrifuged urine, with a threshold of at least 1 Gram-negative rod in 10 oil immersion fields, has greater sensitivity, specificity, and positive predictive value than does the standard urinalysis and is the preferred method of urinalysis when appropriate equipment and personnel are available. (11).

Table 2. shows number of colonies/ml urine (17)

\begin{tabular}{ll}
\hline Negative & $\mathbf{< 1 0 , 0 0 0}$ colonies $/ \mathbf{m l}$ \\
\hline Intermediate & $10,000-100,000$ colonies $/ \mathrm{ml}$ \\
Positive & $>100,000$ colonies $/ \mathrm{ml}$ \\
\hline
\end{tabular}

\subsubsection{Urine Culture}

Culture of the specimen: This procedure is usually performed on plates of 5\% sheep blood agar, which detects growth of most organisms, and on a plate of MacConkey agar or other selective and differential medium for isolation of gram-negative organisms. Additionally, some labs plate urine on colistin-nalidixic acid agar (CNA) or other selective medium for gram-positive bacteria. The plates are incubated at $36^{\circ} \mathrm{C}$ for 18 to 24 hours and read for growth (6).

The isolated microorganisms were identified by colony morphology, Gram stain as well as biochemical and enzymatic reactions including catalase, urease, coagulase, oxidase, bile solubility, carbohydrate fermentation reactions, amino acid decarboxylation on triple sugar iron agar (TSI), lysine iron agar (LIA) and motility indole ornithine (MIO) according to standard methods (8).

A urine culture is a test to detect and identify organisms (usually bacteria) that causing a urinary tract infection (UTI). Urine culture results are usually ready in 1 to 3 days. The urine must be cultured within one hour of collection if not refrigerated. However, refrigerated samples may be stored for up to 24 hours before plating the sample. Urine culture is a quantitative procedure. A calibrated inoculating loop that holds 0.01 or $0.001 \mathrm{~mL}$ of urine is inserted vertically into the urine sample and used to transfer the urine to a sterile agar plate. If urine is obtained by bladder puncture, $0.1 \mathrm{~mL}$ is transferred to the plate using a sterile pipet (5).

\subsection{Statistical Analysis}

Results were statistically analyzed by statistical package SPSS version 16. Two types of statistics were done Descriptive: Analytical:-One a way ANOVA (F test) Kruskal -Wallis test: Post hoc test: Chi-Squared Fisher's exact test: Mann-Whitney test: Pearson's Correlation analysis: $\mathrm{P}$ value significant difference if $\mathrm{P}<0.05$ highly significant difference if $\mathrm{P}<0.001$

\section{Results}

This table shows the demographic distribution of the studied group as follow: the number of our patients was 200 child, (91) male and (109) female. We classify them according to age into three groups. 1-4 years: 81 cases (40.5\%), 4-9 years: 87 cases $(43.5 \%)$ and $9-14$ years: 32 cases $(16 \%)$.

Table 3. shows the demographic data of the studied children.

\begin{tabular}{lll}
\hline & NO & $\%$ \\
\hline Sex & & \\
Male & 91 & $45.5 \%$ \\
Female & 109 & $54.5 \%$ \\
Age & & \\
$1-4$ years & 81 & $40.5 \%$ \\
$>4-9$ years & 87 & $43.5 \%$ \\
$>9-14$ years & 32 & $16.0 \%$ \\
Total & 200 & $100 \%$ \\
\hline
\end{tabular}

This table shows the distribution of results of urine culture. We reported that 107 cases had negative urine culture $(53.5 \%)$, while 93 cases had positive urine culture $(46.5 \%)$.

Table 4. shows the distribution of the results of urine culture in our patients.

\begin{tabular}{llll}
\hline & & NO & $\%$ \\
\hline \multirow{2}{*}{ Urine culture } & $(-\mathrm{VE})$ & 107 & 53.5 \\
& $(+\mathrm{VE})$ & 93 & 46.5 \\
\multirow{2}{*}{ Total } & & 200 & 100 \\
\hline
\end{tabular}

This table shows the sensitivity, specificity, NPV, PPV, True positive, true negative, false positive and false negative for each test controlled by urine culture. We found that the sensitivity and specificity of pus cell were $87 \%$ and $96 \%$, while LE and/or nitrite were $91 \%$ and $90 \%$, BCC sensitivity and specificity were $17 \%$ and $98 \%$. RBCs sensitivity was $10 \%$, while specificity was $99 \%$.

Table 5. This table shows the sensitivity, specificity, NPV, PPV, True positive, true negative, false positive and false negative for each test controlled by urine culture.

\begin{tabular}{|c|c|c|c|c|c|c|c|c|}
\hline \multirow[t]{2}{*}{ The test } & $\begin{array}{l}\text { Sensitivity } \\
(95 \% \text { CI })\end{array}$ & $\begin{array}{l}\text { Specificity } \\
\text { (95\% CI) }\end{array}$ & $\begin{array}{l}\text { PPV } \\
(95 \% \text { CI }) \\
\end{array}$ & $\begin{array}{l}\text { NPV } \\
(95 \% \text { CI })\end{array}$ & $\begin{array}{l}\text { True } \\
\text { positives }\end{array}$ & $\begin{array}{l}\text { False } \\
\text { positives }\end{array}$ & $\begin{array}{l}\text { False } \\
\text { negatives }\end{array}$ & $\begin{array}{l}\text { True } \\
\text { negatives }\end{array}$ \\
\hline & $\%$ & $\%$ & $\%$ & $\%$ & NO. & NO. & NO. & NO \\
\hline Age & $74[.64-.82]$ & $53[.43-63]$ & $58[.49-.67]$ & $70[.59-.82]$ & 69 & 50 & 24 & 57 \\
\hline $\begin{array}{l}<4-9 \text { years } \\
>9-14 \text { years }\end{array}$ & $25[.17-.35]$ & $92[.84-.96]$ & $72[.53-.86]$ & $58[.50-.66]$ & 23 & 9 & 70 & 98 \\
\hline
\end{tabular}




\begin{tabular}{|c|c|c|c|c|c|c|c|c|}
\hline The test & $\begin{array}{l}\text { Sensitivity } \\
(95 \% \text { CI })\end{array}$ & $\begin{array}{l}\text { Specificity } \\
\text { (95\% CI) }\end{array}$ & $\begin{array}{l}\text { PPV } \\
(95 \% \text { CI }) \\
\end{array}$ & $\begin{array}{l}\text { NPV } \\
(95 \% \text { CI })\end{array}$ & $\begin{array}{l}\begin{array}{l}\text { True } \\
\text { positives }\end{array} \\
\end{array}$ & $\begin{array}{l}\text { False } \\
\text { positives }\end{array}$ & $\begin{array}{l}\text { False } \\
\text { negatives }\end{array}$ & $\begin{array}{l}\text { True } \\
\text { negatives }\end{array}$ \\
\hline & $\%$ & $\%$ & $\%$ & $\%$ & NO. & NO. & NO. & NO \\
\hline $\begin{array}{l}\mathrm{RBCs} \\
>10\end{array}$ & $10[0.05-0.18]$ & 99 [0.94- 1.00] & 90 [0.54-0.99] & 56 [0.48-0.63] & 9 & 1 & 84 & 106 \\
\hline $\begin{array}{l}\text { Bacterial colony count } \\
>100.000\end{array}$ & $17[0.10-0.27]$ & 98 [0.93-1.00] & 89 [0.64- 0.98] & 58 [0.50-0.65] & 16 & 22 & 77 & 105 \\
\hline $\begin{array}{l}\text { Pus cells } \\
>10\end{array}$ & $87[0.78-0.93]$ & $96[0.90-.99]$ & $95[0.88-.98]$ & $90[0.82-0.94]$ & 81 & 4 & 12 & 103 \\
\hline Nitrates & $30[0.21-0.41]$ & $92[0.84-0.96]$ & $76[0.58-0.88]$ & $60[0.52-0.68]$ & 28 & 9 & 65 & 98 \\
\hline Leukocyte estrate & $87[0.78-0.93]$ & 94 [0.88-0.98] & 93 [0.85-0.97] & 89 [0.82-0.94] & 81 & 6 & 12 & 101 \\
\hline LE and or nitrates & 91 [0.83-0.96] & $90[0.82-0.95]$ & $89[0.80-0.94]$ & $92[0.85-0.96]$ & 85 & 11 & 8 & 96 \\
\hline
\end{tabular}

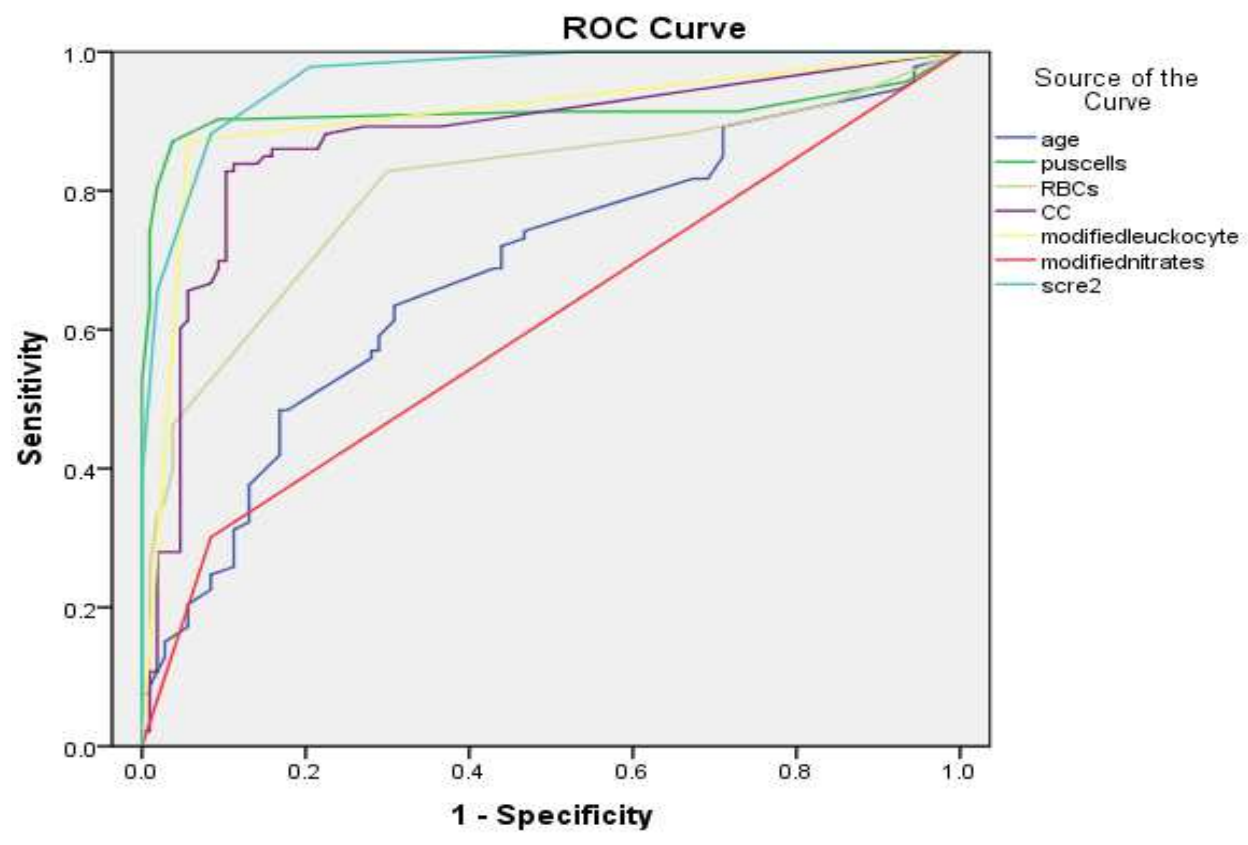

Diagonal segments are produced by ties.

Figure 1. Receiver-Operating Characteristic (ROC) curves

This table shows sensitivity, specificity, Accuracy, PPV, NPV of each cut off point, and we found that the cutoff point (3) had the highest sensitivity (88\%), specificity (92\%), Accuracy (90\%), PPV (90\%), and NPV (90\%).

Table 6. shows sensitivity, specificity, Accuracy, PPV, NPV of each cutoff point in relation to urine culture.

\begin{tabular}{llllll}
\hline cutoff point & Sensitivity & Specificity & Accuracy & PPV & NPV \\
\hline 1 & $99 \%$ & $47 \%$ & $71 \%$ & $62 \%$ & $98 \%$ \\
2 & $89 \%$ & $79 \%$ & $88 \%$ & $81 \%$ & $98 \%$ \\
3 & $88 \%$ & $92 \%$ & $90 \%$ & $90 \%$ & $90 \%$ \\
4 & $66 \%$ & $98 \%$ & $83 \%$ & $97 \%$ & $77 \%$ \\
5 & $39 \%$ & $99 \%$ & $71 \%$ & $97 \%$ & $65 \%$ \\
6 & $17 \%$ & $99 \%$ & $61 \%$ & $94 \%$ & $58 \%$ \\
7 & $2 \%$ & $99 \%$ & $54 \%$ & $67 \%$ & $54 \%$ \\
\hline
\end{tabular}

\section{Discussion}

UTIs are the most common bacterial infections in infants and young children, and can be associated with high fever and poor general conditions (23).

The infection may involve the kidney, and in a small proportion of patients even lead to permanent renal damage and scarring (14).

Culture of urine is the reference standard for the diagnosis of UTI (23), but continuing efforts are spent in searching for alternative, more rapid and still accurate tests. This is especially important in children, since both a delay in treatment and the inappropriate administration of antibiotics can result in potentially serious adverse effects (21).

In this study 200 child 1-15yr 91 males (45\%) and 109 females (54\%) attending in Berket Al Sabaa Hospital complaining of urinary tract symptoms as (frequency, dysuria, fever, and suprapubic pain) and had no antibiotics for 3 days before collection of samples screened for UTI by complete urine analysis, dipstick and bacterial colony count then confirmed by urine culture.

In our study urinary tract infection was prevalent in females than males where 57 females were positive cases $(61.3 \%)$, while 36 males were positive cases $(38.7 \%)$.

That result agreed also with Muoneke et al., 2012 who made a study which was a study conducted at the Children's Emergency Unit and the Children's Outpatient Department of Ebonyi State University Teaching Hospital, Abakaliki, 
Ebonyi State,Nigeria. About one hundred and ten patient that attended to that center proved to have UTI. The positive cases were $48(42.8 \%)$ males and $64(57.2 \%)$ females.

In contrary with our result, Rosa Luciano et al., 2011 made a study which was carried out at the Bambino Gesu Children's Hospital, a tertiary pediatric hospital and clinical research institute in Roma, Italy. We retrospectively analyzed the data of 852 consecutive pediatric patients $(\leq 14$ years of age) assessed for UTI in three periods: March-September 2006, January-April 2007, and November-December 2007. Both hospitalized patients, and children referred to the outpatient clinic of the Laboratory Unit were included. That study showed that 32 female (13.9\%) and 37 male $(15.4 \%)$ had positive urine culture, while 176 female $(84.6 \%)$ and 229 male $(86.1 \%)$ had negative urine culture.

In our study, according to age were 81 cases $(40.5 \%)$ were $1-4$ years, 87 cases $(43.5 \%)$ were $>9-14$ years, 32 cases $(16 \%)$ were $>4-9$ years. In 1-4 years age group 24 cases $(29.6 \%)$ had positive urine culture, while 57 cases $(70.4 \%)$ had negative urine culture. In 4-9 years age group 46 cases (52.9\%) had positive urine culture, while 41 cases $(47.1 \%)$ had negative urine culture. In $>9-14$ years age group 23 cases (71.9\%) had positive urine culture, while 9 cases $(28.1 \%)$ had negative urine culture.

Rosa Luciano et al., 2011 found in her study that positive cases from 1-4 years were $18(13.1 \%)$ while negative cases $119(86.9 \%)$, positive cases from 5-9 years were $23(12.4 \%)$ while negative cases $136(87.6 \%)$, and positive cases from $10-14$ years were $8(8.3 \%)$ while negative cases $89(91.8 \%)$.

In our study, the most common symptoms of urinary tract infection were frequency $(46.4 \%)$ then dysurea (40.3\%), suprapubic pain $(8.6 \%)$ and fever $(4.7 \%)$.

Muoneke et al., 2012 disagreed with our results whose found that most common symptoms were fever (76.4\%), vomiting (17.3\%). diarrhea $(16.4 \%)$, abdominal pains $(11.8 \%)$, body/leg swelling $(10 \%)$, hematuria $(8.1 \%)$, dysuria $(5.5 \%)$, frequency $(3.6 \%)$, weight loss $(2.7 \%)$, flank pains $(2.7 \%)$, convulsion $(2.7 \%)$ and headache $(1.8 \%)$, but this study included patients less than 2 years (younger than patients included in our study).

In our study we found that pus cells in relation to urine culture is valid in diagnosis of cases of UTI, where its Sensitivity was $87 \%$, specificity was $96 \%$, PPV was $95 \%$, and NPV was $90 \%$.

Rosa Luciano et al., 2011 found that validity of pus cells in relation to urine culture in diagnosis of cases of UTI, Sensitivity was (30.4\%), specificity was (94.6), PPV was $(48.8 \%)$, and NPV was $(88.9 \%)$.

Kathy et al., 2001study agreed with our study and reported the sensitivity $(82 \%)$ and specificity $(87 \%)$ of pus cell in relation of urine culture.

However American academy of pediatrics, 2011, reported thatthe sensitivity and specificity of pus cell in relation to urine culture among patients with symptoms of urinary tract infection was $(73 \%)$ and $(81 \%)$.

Maria Bitsori and Emmanouil, 2012 study found that sensitivity of pus cell (>5/hpf) was $(73 \%)$, and the specificity was $(81 \%)$

According to our study, the sensitivity of RBCs in urine was $(10 \%)$ and the specificity was $99 \%$, PPV was $(90 \%)$ and NPV was (56\%) that disagreed with Brett White. 2011 study which reported $(47 \%)$ sensitivity, $(78 \%)$ specificity.

Other study like Rosa Luciano et al., 2011 study agreed with our study which found that the sensitivity of RBCs in urine was $(10.1 \%)$ and the specificity was $(96.3 \%)$, PPV was $(31.8 \%)$ and NPV was $(86.3 \%)$.

According to our study, leukocyte esterase (LE) sensitivity was $(87 \%)$, specificity was $(94 \%)$, positive predictive value (PPV) was (93\%), negative predictive value (NPV) was $(89 \%)$, and nitrite sensitivity is $(30 \%)$, specificity was $(92 \%)$, PPV was (93\%), NPV was (89\%), while leukocyte esterase (LE) and nitrite sensitivity was $(91 \%)$, specificity was( $90 \%)$, PPV was (89\%) and NPV was (92\%).

Rosa Luciano et al., 2011 study found that leukocyte esterase (LE) sensitivity was $(49.3 \%)$, specificity was (87.9\%), positive predictive value (PPV) was (43\%), negative predictive value (NPV) was $(91.1 \%)$, and nitrite sensitivity is (18.8\%), specificity was (100\%), PPV was (100\%), NPV was $(87.9 \%)$, while leukocyte esterase (LE) and nitrite sensitivity was (53.6\%), specificity was( $87.9 \%), P P V$ was $(43 \%)$ and NPV was $(91.8 \%)$.

Yehezkel et al., 1999 study reported that leukocyte esterase (LE) and nitrite sensitivity was $(97.1 \%)$, specificity is $(82.5 \%)$, PPV is (69.4\%) and NPV is (98.6\%).

In our study cases that had bacterial colony count from 0 104 were 112 cases (56\%), from 104- 105 were 72 cases (36\%), and more than 105 were 16 cases $(16 \%)$.

Our study showed that sensitivity of bacterial colony count more than 105 was $(17 \%)$, while the specificity was $(98 \%)$, PPV was (89\%), and NPV was (58\%).

Rosa Luciano et al., 2011 study used Microscope IRIS IQ200 to detect bacterial count, and found the sensitivity was $(24.6 \%)$, specificity is (97.3\%), PPV is $(60.7 \%)$ and NPV is $(88.3 \%)$ and that agreed with our study.

In our study all 200 cases had urine culture, from them 93 cases were positive while 107 cases were negative. The positive cases were 36 male and 57 female, while the negative cases were 55 male and 52 female.

In our study we did a scoring system for our children with urinary tract infection depending on their results. We choose a cut-off point $>3$ because its sensitivity was (88\%), specificity is (92\%), PPV is (90\%) and NPV is (90\%).

Through the selection of different cut-off criteria, the score can be adapted to different clinical situations depending on the relative benefits of maximizing sensitivity or specificity. The selection of a very low cut-off, basically corresponding to any of the variables included in the final model being positive, leads to $88 \%$ sensitivity and $90 \%$ negative predictive value, allowing withholding treatment in children scored negative pending culture results. In our series of 200 children with suspected UTI, such a procedure would avoid unnecessary antibiotic administration in 109 children. A higher cut-off (e.g. $\geq 4$ ) leads to $98 \%$ specificity, increasing the positive predictive value to $97 \%$ without large loss of 
NPV (77\%). The use of this strategy would avoid unnecessary treatment in many non-infected children.

Rosa Luciano et al., 2011study agreed with our study which included 474 children with suspected UTI as such a procedure avoided unnecessary antibiotic administration in 292 children. In this study they selected a very low cut-off, basically corresponding to any of the variables included in the final model being positive, leads to $73.9 \%$ sensitivity and $94.2 \%$ negative predictive value. A higher cut-off (e.g. $\geq 4$ ) leads to $96.3 \%$ specificity, increasing the positive predictive value to $69.4 \%$ without large loss of NPV (91.8\%).

So our conclusion is patient who takes a score 3 or more should begin treatment before urine culture appear, while who takes a score less than 3 should not begin treatment until confirmatory urine culture appear.

\section{References}

[1] American Academy of Pediatrics (AAP) (2011): Urinary Tract Infection: Clinical Practice Guideline for the Diagnosis and Management of the Initial UTI in Febrile Infants and Children 2 to 24 Months. Pediatrics; 61(11):56-98.

[2] Bauer R, and Kogan BA. (2008): New developments in the diagnosis and management of pediatric ITO. UrolClin North Am; (35): 47 - 85.

[3] Bhat R.G, Katy T.A. and Place F.C.(2011): Pediatric urinary tract infections.Emerg Med Clin N Am, 29, pp. 637-653.

[4] Brett White. (2011): Diagnosis and Treatment of Urinary Tract Infections in Children Oregon Health and Science University, Portland, Oregon Am Fam Physician. Feb 15; 83(4):409-415.

[5] Chernecky CC, Ritchie G, and Berger BJ (2008): Laboratory Tests and diagnostic procedures (5th ed.). Philadelphia, PA: Elsevier Saunders. Clin. Chem: 7(6); 603:12-20.

[6] Chernecky, Cynthia C, Berger J,et al., (2001):Laboratory Tests and Diagnostic Procedures, 3rd ed. Philadelphia, PA: W. B. Saunders Company. Clin. Chem: 7(5); 211:12-3.

[7] Heffner, V., Gorelick, M (2008): Pediatric Urinary Tract Infection. ClinPedEmerg Med.; 9: 233-237.

[8] Henry JB, Davey FR, Herman CJ, et al.,(2001):editors. Basic Laboratory Tests, Mosby Elsevier management by laboratory methods. 20th ed. ;99(8):367-403.

[9] John, SA. et al., (2006): The use of urinary dipstick test to exclude urinary tract infection: a systematic review of the literature, American Journal of clinical pathology; 126 (3): 428-36.
[10] Kathy N. Shaw, Karin L. et al., (2001): Screening for urinary tract infection in infants in the emergency department: which test is best. Pediatrics; 101: 1 .

[11] Kenneth B., and Roberts M.D. (2011): Urinary Tract Infection: Clinical Practice Guideline for the Diagnosis and Management of the Initial UTI in Febrile Infants and Children 2 to 24 Months. PEDIATRICS ; $128-3$

[12] Mangin D. (2010): Urinary tract infection in primary care. BMJ, 340, p. 657.

[13] Maria Bitsori and Emmanouil G. (2012): Pediatric Urinary Tract Infections Expert Rev Anti Infect Ther.; 10(10):11531164.

[14] Montini G., Tullus K. and Hewitt I. (2011): Febrile urinary tract infections in children NEJM, 365; 239-250.

[15] Muoneke V.U., Ibekwe M.U. and Ibekwe R.C. (2012): Annals of Medical and Health Sciences Research, Childhood urinary tract infection in Abakaliki: Etiological organisms and antibiotic sensitivity $2 ; 1: 29-32$.

[16] National Institute for Health and Clinical Excellence (NICE). (2007): Urinary tract infection in children: diagnosis,treatment and long-term management. NICE clinical guideline54. Available: http:// www.nice.org.uk/cg054.

[17] Pagana KD and Pagana TJ (2010): Mosby's Manual of Diagnostic and Laboratory Tests, 4th ed. St. Louis: Mosby Elsevier, 700-701.

[18] Rosa L., Simone P., Leonardo F., Marta A., Francesca F., Marina C., Emilia M., Francesco E. and Maurizio M. (2011): Development of a score based on urinalysis to improve the management of urinary tract infection in children. ClinicaChimicaActa Volume 413: 3-4, 18 February, 478-482.

[19] Stanley R, Pagon Z and Bachur R. (2005): Hyperpyrexia among infants younger than 3 months.Pediatric Emergency, 21(5):291-4.

[20] Tanagho, Emil A., Mcaninch, Jack W., et al., (2004): United States of America: McGraw-Hill companies Inc;. Bacterial Infections of the genitourinary tract p 203-227.

[21] Williams G.J., Macaskill P., Chan S.F., Turner R.M. , Hodson E. and Craig J.C. (2010): Absolute and relative accuracy of rapid urine tests for urinary tract infection in children: a metaanalysis Lancet Infect Dis, 10 , pp. 240-250.

[22] Yehezkel W., Elisheva Z., Lisa A. and Marc M. (1999):The Validity of the Uriscreen Test for Early Detection of Urinary Tract Infection in ChildrenPEDIATRICS 104 No. 4 October.

[23] Zorc J.J., Kiddoo D.A. and Shaw K.N. (2005): Diagnosis and management of pediatric urinary tract infectionsClinMicrobiol Rev, 18, 417-422. 